\title{
Bimodulus bending model for bamboo poles
}

Rodolfo Lorenzo ${ }^{a^{*}}$, Leonel Mimendia, Haitao Li ${ }^{\mathrm{b}}$, Dong Yang ${ }^{\mathrm{b}}$

a Department of Civil, Environmental and Geomatic Engineering, University College London, London WC1E 6BT, UK

${ }^{b}$ College of Civil Engineering, Nanjing Forestry University, Nanjing 210037, China

*Corresponding author: r.lorenzo@ucl.ac.uk (R. Lorenzo)

\section{Abstract}

The building industry is currently under pressure to transit from non-renewable materials with high embodied energy towards natural and sustainable options. Bamboo poles are some of the most promising renewable structural elements, but their formal utilisation in construction requires the development of appropriate analytical models and new design tools to address their challenging natural and organic nature. Considering the composite nature of bamboo poles and the properties of their high-strength sclerenchyma fibres and parenchyma matrix, this paper presents the formulation of an analytical bimodulus model to determine the cross-sectional strain and stress distribution in bamboo poles in bending. This model was validated through fullscale experimental four-point bending tests on Moso (Phyllostachys pubescens) bamboo poles suggesting a constant tensile elastic modulus of fibres for this species.

\section{Keywords}

Bamboo; Analytical modelling; Mechanical testing; Bending

\section{Introduction}

The world's increasing urbanisation is placing an unprecedented burden on natural resources and the environment. By 2017, the construction industry was responsible for the largest global share of energy consumption and carbon dioxide emissions accounting for $36 \%$ and $39 \%$ respectively [1]. Global guidelines have been introduced to reduce the negative impact of the building industry in cities by increasing the efficiency of current building procedures and materials [2]. Nonetheless, a gradual transition from non-renewable and highly industrialised materials to natural, sustainable and renewable ones is essential to achieve a balanced sustainable development. This transition, however, requires the parallel development of new analytical models and design tools that take into account the distinctive features of these natural 
materials as opposed to simply adopting current models and tools which have been mostly ineffective in supporting their formal use in construction.

Bamboo poles are natural high-strength fibre reinforced structural elements which have been largely used to develop local, self-build housing projects in developing countries but more recently this material has been considered an important component of the United Nations' Sustainable Development Goal 12 due to its renewable, sustainable and resilient nature [3]. Compared with wood, bamboo poles are a fast-growing and finished structural element with a much faster maturing period of three to four years which is approximately half that of any softwood $[4,5]$. The carbon sequestration capacity of bamboo is similar to that of tropical forests, with a well-managed plantation capable of generating negative environmental footprints [6-8]. In terms of sustainable construction, bamboo is twenty times more favourable than wood, metals or masonry products when locally sourced [9]. While the environmental credentials of bamboo poles make them a fitting alternative construction material, technical engineering challenges remain and need significant efforts to address them and support the formal adoption of bamboo poles as a building element.

One of these challenges is related to the natural variability of the mechanical properties found not only across different species, but even within single bamboo poles due to the changes in microstructure along their length [10-12]. In order to manage this variability, new efficient methods for intensive fabrication and mechanical testing have been proposed [13]. These methods are intended to increase our basic long-term understanding of the material while providing the basic information for the formal analysis and assessment of bamboo structures in the short-term through simple analytical models specific for this material such as the one proposed in this work. Bamboo poles are a natural composite material [14] with a relatively simple anatomical structure of unidirectional vascular bundles consisting of high-strength sclerenchyma fibres and conducting vessels oriented along the length of the pole and embedded in a matrix of parenchyma cells $[5,15]$. The unpredictable organic nature of these constituents, whose development is in turn influenced by external factors during growth, is partly responsible for the inherent variability found in the mechanical properties of bamboo poles [1416]. The current international standard for bamboo construction ISO 22157:2019 [17] specifies 
the adoption of full-scale experimental testing to determine the bending properties of bamboo poles. These full-scale bending tests are usually difficult to implement, and their corresponding results formulation is based on standard bending theory that neglects the composite nature of the material. Previous research focused on the study of the tensile and compressive behaviour of bamboo and its constituent elements are based on much simpler experimental tests on small clear bamboo samples and have confirmed the significantly different tensile and compressive elastic properties of bamboo [14,18-20]. As such, an analytical bimodulus model is required to determine the cross-sectional stress distribution of bamboo poles in bending. Bimodulus materials were first introduced by [21] and later applied to fibre-reinforced composites with their constituent materials having different elastic moduli in compression and tension [22-24].

This work presents the development of an analytical bending model considering the fundamental principles of unidirectional composite materials based on simple experimental compression tests of small clear bamboo samples and basic imaging processing to determine the volume fraction of fibres. This analytical model is validated through full-scale, experimental four-point bending tests on Moso (Phyllostachys pubescens) bamboo poles.

\section{Analytical model}

Bamboo is a natural composite material and so the contribution of its constituent materials, matrix and fibres, to resist tensile and compressive stresses will vary as a function of their relative stiffnesses under each of these stresses. The tensile stiffness of bamboo fibres is a couple of magnitudes higher than the comparatively flexible matrix [14] and therefore this model neglects the contribution of the matrix to the tensile stiffness of bamboo in bending. On the contrary, a comparable contribution from both fibres and matrix acting compositely is considered for the compressive stiffness of the poles. As such, the proposed bimodulus bending model is based on the use of the elastic modulus of fibres for the tensile region of the cross section and the combined elastic modulus of both matrix and fibres for the compression region. The cross-sectional fibre content is assumed constant and equal to the average value across the bamboo wall as the effect of the fibre gradient is considered negligible for thin poles with a diameter:thickness ratio greater than approximately $8[11,12]$. The effect of the fibre gradient on thick poles is beyond the scope of this work. 
This model assumes a linear elastic material and follows the principles of Euler-Bernoulli beam theory [25]. Geometrically, bamboo poles in this study are assumed to be uniform circular tubes following the traditional idealisation adopted for bamboo poles [17]. The effect of this idealisation on the behaviour of poles with a significant geometric variability is beyond the scope of this research.

Figure 1 shows a bamboo cross section idealised as a thin ring of average radius, $\bar{R}$ and thickness $t$ together with its linear strain and stress distribution in bending. The bilinear stress distribution assumes two different elastic moduli namely, $E_{c}$ in compression (matrix and fibres) and $E_{t}$ in tension (fibres only). The distance $\bar{y}$ from the extreme fibre in tension to the neutral axis defined in terms of the angle $\theta$ shown in Figure 1 is given by:

$\bar{y}=\bar{R}(1-\cos \theta)$

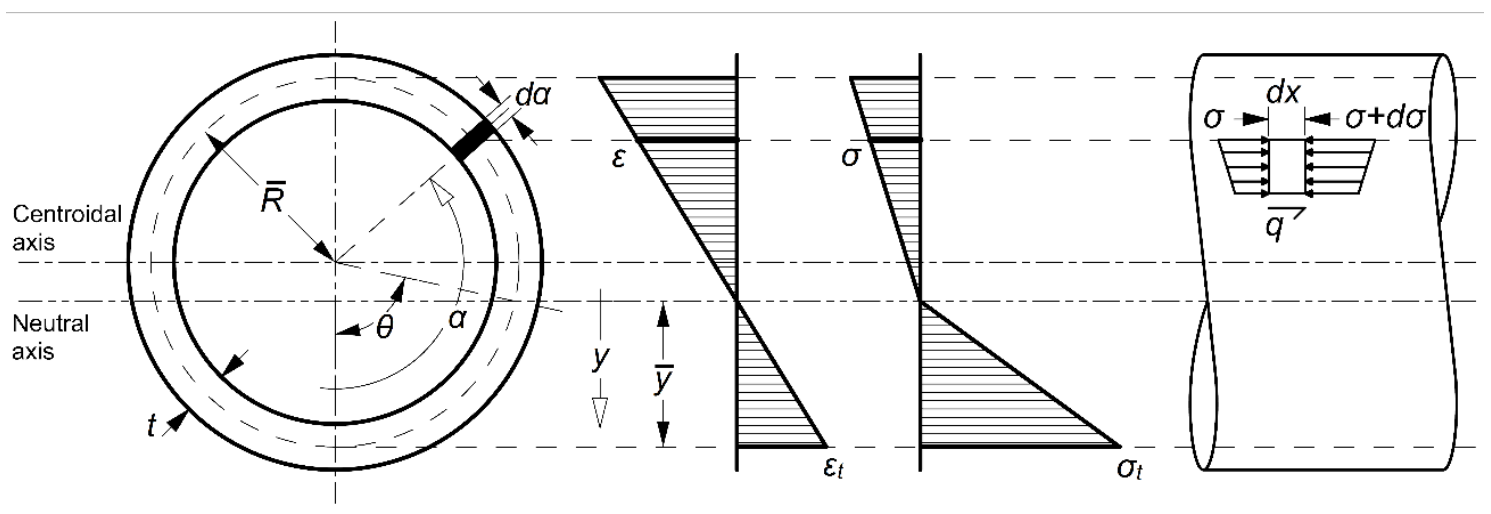

Figure 1. Bamboo cross section and corresponding strain and stress distribution in bending From compatibility of deformations and Equation (1), the strain, $\varepsilon$, within the section is defined by:

$\varepsilon=\frac{\varepsilon_{t}}{\bar{y}} y=\frac{\varepsilon_{t}}{\bar{R}(1-\cos \theta)} y$

where $\varepsilon_{t}$ is the tensile strain at the section extreme fibre and $y$, expressed as a function of the angles $\theta$ and $\propto$ shown in Figure 1, is given by:

$y=\bar{R}(\cos \alpha-\cos \theta) ; 0 \leq \alpha \leq \pi$

Substituting Equation (3) in (2) gives:

$\varepsilon=\varepsilon_{t} \frac{\cos \alpha-\cos \theta}{1-\cos \theta} ; 0 \leq \alpha \leq \pi$

From Hooke's law [25], the tensile and compressive forces, $d F_{t}$ and $d F_{c}$, acting on an infinitesimal sector of tube are: 
$d F_{t}=E_{t} \varepsilon d A$

$d F_{c}=E_{c} \varepsilon d A$

where:

$d A=\left\{\begin{array}{c}t \bar{R} v_{f} d \alpha ; 0 \leq \alpha \leq \theta \\ t \bar{R} d \alpha ; \theta<\alpha \leq \pi\end{array}\right.$,

and the volume fraction, $v_{f}$, of the cross section is defined as the ratio between the area of fibres, $A_{f}$, and its total area, $A$ :

$v_{f}=\frac{A_{f}}{A}$

Defining the modular ratio, $n$, as:

$n=\frac{E_{c}}{E_{t}}$

and integrating equations (5) and (6) leads to the total tensile and compressive forces, $F_{t}$ and $F_{c}$, acting on the section:

$F_{t}=\frac{E_{t} \varepsilon_{t} v_{f} t \bar{R}}{1-\cos \theta}\left[2 \int_{0}^{\theta}(\cos \alpha-\cos \theta) d \alpha\right]=2 E_{t} \varepsilon_{t} v_{f} t \bar{R} \frac{\sin \theta-\theta \cos \theta}{1-\cos \theta}$

$F_{c}=\frac{n E_{t} \varepsilon_{t} t \bar{R}}{1-\cos \theta}\left[2 \int_{\theta}^{\pi}(\cos \alpha-\cos \theta) d \alpha\right]=2 n E_{t} \varepsilon_{t} t \bar{R} \frac{(\theta-\pi) \cos \theta-\sin \theta}{1-\cos \theta}$

From equilibrium:

$F_{t}+F_{c}=0$

and substituting Equations (10) and (11) in (12) leads to:

$\left(v_{f}-n\right)(\tan \theta-\theta)-n \pi=0$

which defines the position of the neutral axis (i.e. angle $\theta$ ) as a function of both the volume fraction, $v_{f}$, and modular ratio, $n$ of the section.

The bending moment acting on the section is calculated from the product of the tensile and compressive forces in Equations (5) and (6) and their corresponding distances to the centroidal axis:

$$
\begin{aligned}
M & =2 \int_{0}^{\theta}(\bar{R} \cos \alpha) d F_{t}+2 \int_{\theta}^{\pi}(\bar{R} \cos \alpha) d F_{c}= \\
& =\frac{2 t \bar{R}^{2} E_{t} \varepsilon_{t}}{1-\cos \theta}\left[v_{f} \int_{0}^{\theta}\left(\cos ^{2} \alpha-\cos \alpha \cos \theta\right) d \alpha+n \int_{\theta}^{\pi}\left(\cos ^{2} \alpha-\cos \alpha \cos \theta\right) d \alpha\right]
\end{aligned}
$$

Integrating and simplifying Equation (14) leads to:

$M=\frac{I}{\bar{y}} E_{e q} \varepsilon_{t}$ 
where $I$ is the second moment of area of a thin circular tube:

$I=\pi t \bar{R}^{3}$

The equivalent elastic modulus of bamboo, $E_{\text {eq }}$, provides a single value for the bending stiffness of the section and is defined as:

$E_{e q}=K E_{t}$

with the factor $K$ given by:

$K=\frac{1}{\pi}\left[\left(n-v_{f}\right) \frac{\sin 2 \theta}{2}+\left(v_{f}-n\right) \theta+n \pi\right]$

The bending stresses, $\sigma$, within the section are defined by:

$\sigma=\left\{\begin{array}{c}E_{t} \varepsilon=E_{t} \varepsilon_{t} \frac{\cos \alpha-\cos \theta}{1-\cos \theta} ; 0 \leq \alpha \leq \theta \\ E_{c} \varepsilon=n E_{t} \varepsilon_{t} \frac{\cos \alpha-\cos \theta}{1-\cos \theta} ; \theta<\alpha \leq \pi\end{array}\right.$

Rearranging Equation (15):

$E_{t} \varepsilon_{t}=\frac{M \bar{y}}{I K}$

and substituting Equation (20) in (19) gives the general expression for the bending stress

distribution within the section:

$\sigma=\left\{\begin{array}{c}\frac{M y}{I K}=\frac{M \bar{R}}{I K}(\cos \alpha-\cos \theta) ; 0 \leq \alpha \leq \theta \\ \frac{n M y}{I K}=\frac{n M \bar{R}}{I K}(\cos \alpha-\cos \theta) ; \theta<\alpha \leq \pi\end{array}\right.$

Also, from equilibrium of horizontal forces in Figure 1:

$\int \sigma d A+q d x-\int(\sigma+d \sigma) d A=0$

and thus, the shear flow, $q$, is defined by:

$q=\int \frac{d \sigma}{d x} d A$

Substituting Equations (7) and (21) in (23):

$q=\left\{\begin{array}{l}\frac{d M / d x}{I K} \int_{0}^{\alpha} \bar{R}(\cos \alpha-\cos \theta) v_{f} t \bar{R} d \alpha ; 0 \leq \alpha \leq \theta \\ \frac{n d M / d x}{I K} \int_{\alpha}^{\pi} \bar{R}(\cos \alpha-\cos \theta) t \bar{R} d \alpha ; \theta<\alpha \leq \pi\end{array}\right.$

and knowing that the shear force, $V$, is defined as [25]:

$V=\frac{d M}{d x}$

leads to: 
$q=\left\{\begin{array}{c}V \frac{v_{f} t \bar{R}^{2}}{I K}(\sin \alpha-\alpha \cos \theta) ; 0 \leq \alpha \leq \theta \\ V \frac{n t \bar{R}^{2}}{I K}[(\alpha-\pi) \cos \theta-\sin \alpha] ; \theta<\alpha \leq \pi\end{array}\right.$

and to the expression for the shear stresses, $\tau$, within the section:

$\tau=\frac{q}{t}$

\section{Model validation}

The proposed bending model for bamboo poles was calibrated against the results of a series of experimental four-point bending tests on 20 bamboo poles comparing the apparent modulus of elasticity in bending, $E_{b}$, obtained from these tests with the equivalent modulus of elasticity, $E_{e q}$, calculated from Equation (17).

\subsection{Experimental data}

\subsubsection{Material}

All 20 bamboo poles were three to four years old Phyllostachys pubescens (Moso) with an average length of $4 \mathrm{~m}$ and diameter of $92 \mathrm{~mm}$ procured from Jiangsu Province, China. The poles were harvested and treated using a carbonisation process in which the poles are placed in a horizontal furnace for 90 minutes at $75{ }^{\circ} \mathrm{C}, 45 \%$ humidity and $1.60 \mathrm{MPa}$ followed by airdrying for 1-2 weeks on well-ventilated racks protected from direct sunlight.

\subsubsection{Apparent modulus of elasticity, $E_{b}$}

The apparent modulus of elasticity in bending, $E_{b}$, of the poles was determined from a series of four-point bending tests as shown in Figure 2. These tests were conducted using a multifunctional structural test system Popwil MAS-300 with a $300 \mathrm{kN}$ actuator and corresponding load cell together with a SICK OD Precision OD5-500W200 laser displacement measurement sensor and data logger TMIL TDS-530. Based on a clear span, $L$, of $3 \mathrm{~m}$ for all poles, $E_{b}$ was calculated as [17]:

$E_{b}=\frac{23 F_{l i n} L^{3}}{1296 I_{B} \Delta_{l i n}}$

where $F_{l i n}$ and $\Delta_{\text {lin }}$ are the maximum applied load and the corresponding mid-span deflection within the linear range of the load-displacement curve determined on the basis of a leastsquares regression with a coefficient of determination $\left(R^{2}\right)$ of 0.99 as shown in Figure 3 for a typical test. The use of a tangent elastic modulus specified in ISO 22157:2019 [17] was not 
considered appropriate for this study due to the significant deflection of the poles (average span:deflection ratio of 150 ) already achieved at $20 \%$ of the ultimate load defined in ISO 22157:2019 as the lower limit of the linear range. The second moment of area, $I_{B}$, is given by:

$I_{B}=\frac{\pi}{4}\left[D^{4}-(D-2 t)^{4}\right]$

where $D$ and $t$ are the average diameter and thickness of the pole calculated according to ISO 22157:2019 [17]. The moisture content of each pole $\left(w_{E_{b}}\right)$ was measured at the time of testing using a portable Delmhorst BD-2100 moisture meter [26].

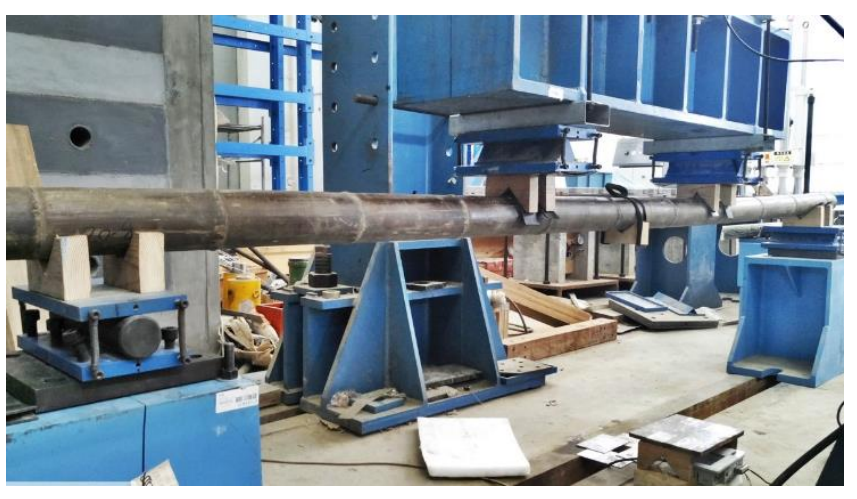

Figure 2. Four-point bending test set-up

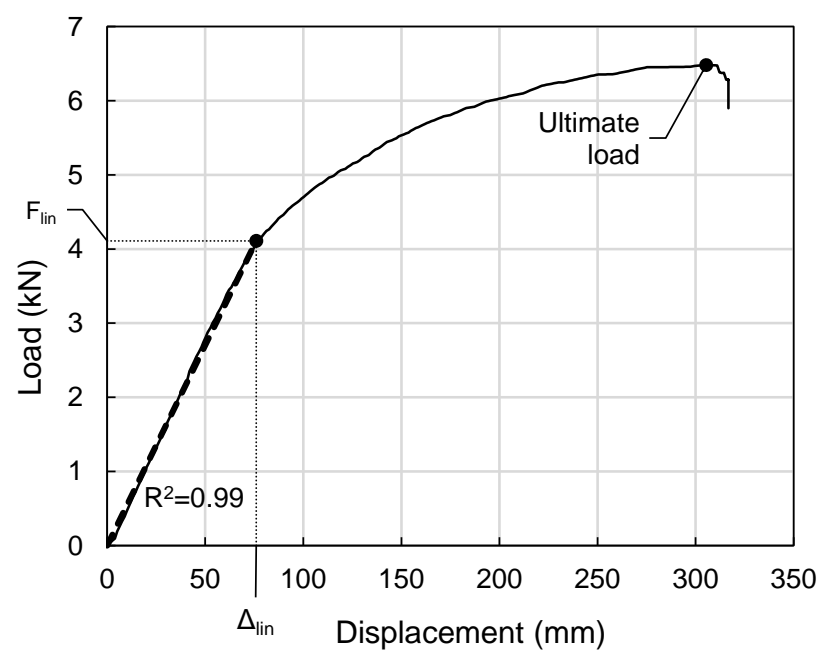

Figure 3. Typical load-displacement diagram and assumed linear range (pole M03) The properties and experimental values of $E_{b}$ for all bamboo poles are shown in Table 1 and Figure 4. 


\begin{tabular}{cccccccc} 
Pole & $\begin{array}{c}\mathbf{D} \\
(\mathrm{mm})\end{array}$ & $\begin{array}{c}\mathbf{t} \\
(\mathrm{mm})\end{array}$ & $\begin{array}{c}\boldsymbol{w}_{\boldsymbol{E b}} \\
(\%)\end{array}$ & $\begin{array}{c}\boldsymbol{E}_{\boldsymbol{b}} \\
(\mathrm{MPa})\end{array}$ & $\begin{array}{c}\boldsymbol{E}_{\boldsymbol{c}} \\
(\mathrm{MPa})\end{array}$ & $\boldsymbol{v f}$ & $\begin{array}{c}\boldsymbol{E}_{\text {eq }} \\
(\mathrm{MPa})\end{array}$ \\
\hline M01 & 95.1 & 8.8 & 15.7 & 11512 & 11169 & 0.29 & 12198 \\
M02 & 94.6 & 8.5 & 15.5 & 13026 & 11633 & 0.35 & 13591 \\
M03 & 90.4 & 9.0 & 13.5 & 13807 & 13205 & 0.32 & 13984 \\
M04 & 84.3 & 9.8 & 13.4 & 13098 & 11567 & 0.30 & 12511 \\
M05 & 92.9 & 7.3 & 14.1 & 11664 & 10072 & 0.33 & 12256 \\
M06 & 90.4 & 8.4 & 14.4 & 10280 & 8835 & 0.31 & 10983 \\
M07 & 95.0 & 7.6 & 12.1 & 13167 & 12354 & 0.35 & 13964 \\
M08 & 88.4 & 8.0 & 13.1 & 11431 & 10288 & 0.32 & 12118 \\
M09 & 88.4 & 8.6 & 14.2 & 14159 & 13374 & 0.31 & 13865 \\
M10 & 82.2 & 6.4 & 18.9 & 12722 & 10735 & 0.37 & 13343 \\
M11 & 95.3 & 6.8 & 14.4 & 13875 & 14627 & 0.35 & 15301 \\
M12 & 83.5 & 9.0 & 15.0 & 12528 & 11104 & 0.35 & 13195 \\
M13 & 94.5 & 8.4 & 13.0 & 10758 & 9275 & 0.31 & 11259 \\
M14 & 95.0 & 7.1 & 13.5 & 12998 & 10963 & 0.35 & 13083 \\
M15 & 87.0 & 7.8 & 12.6 & 13551 & 12775 & 0.35 & 14337 \\
M16 & 99.2 & 6.7 & 10.5 & 13551 & 13727 & 0.35 & 14788 \\
M17 & 96.3 & 7.6 & 10.7 & 15163 & 14735 & 0.34 & 15233 \\
M18 & 91.2 & 9.2 & 10.8 & 12579 & 11162 & 0.32 & 12655 \\
M19 & 89.2 & 9.8 & 10.8 & 13806 & 11757 & 0.33 & 13227 \\
M20 & 99.2 & 7.5 & 12.0 & 13754 & 11996 & 0.34 & 13565
\end{tabular}

Table 1. Summary of analytical and experimental results

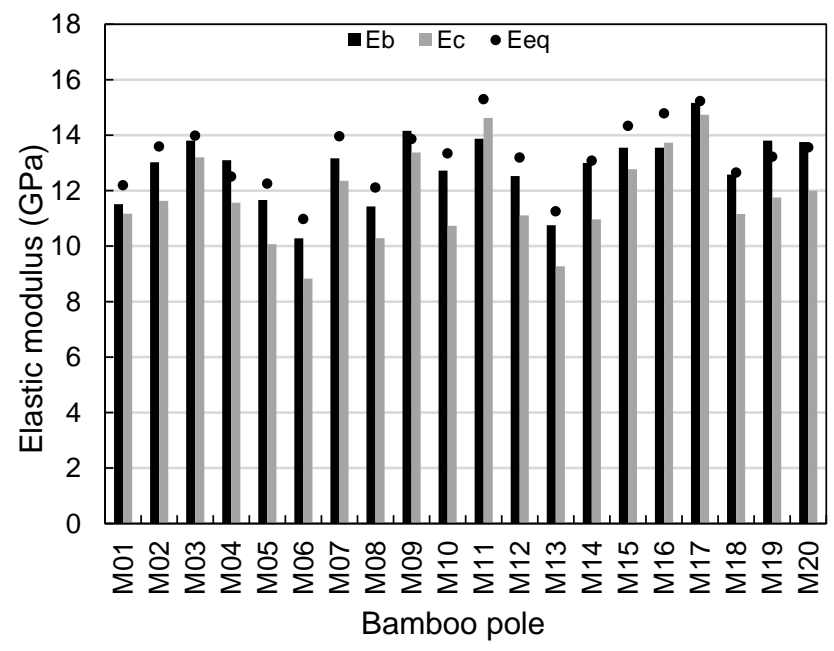

Figure 4. Analytical and experimental elastic moduli

\subsubsection{Compressive elastic modulus, $E_{c}$}

The compressive elastic modulus, $E_{c}$, was determined based on the Chinese industry standard JG/T 199-2007 [27]. Accordingly, two diametrically opposite small clear samples, $60 \mathrm{~mm}$ long $\times$ $15 \mathrm{~mm}$ wide $\times$ wall thickness, were extracted from the internodes at each end of the $4 \mathrm{~m}$-long 
bamboo poles. These samples were kept at a constant temperature of $20^{\circ} \mathrm{C} \pm 2^{\circ} \mathrm{C}$ and relative humidity of $65 \% \pm 5 \%$ for 14 days. The mechanical testing of these samples was carried out using an Instron 3345, 5kN electro-mechanical single-column machine with spherical seat compression platens and an Instron 2630 clip-on strain gauge extensometer. A typical strainstress plot showing the required loading/unloading cycles is shown in Figure 5. The value of $E_{c}$ for each pole was calculated as the average value of all samples extracted from each pole adjusted to the moisture content of the source pole at the time of the four-point bending test in order to allow a direct comparison such that [27]:

$E_{\mathrm{c}}=\frac{1}{n} \sum \frac{K_{E_{\mathrm{c}}}}{K_{E_{b}}} \frac{\Delta \sigma}{\Delta \varepsilon}$

where $n$ is the number of samples (four); $\Delta \sigma$ is the stress difference between the minimum ( 5 $\mathrm{MPa}$ ) and maximum (20 MPa) stress limits and $\Delta \varepsilon$ is the corresponding deformation difference measured at these stress limits. $K_{E_{\mathrm{c}}}$ and $K_{E_{\mathrm{b}}}$ are the correction factors for the effect of moisture content given by [27]:

$K_{E_{\mathrm{c}}}=\frac{1}{0.89+0.36 e^{-0.1 w_{E_{C}}}}$

$K_{E_{\mathrm{b}}}=\frac{1}{0.89+0.36 e^{-0.1 w_{E_{b}}}}$

and $w_{E_{C}}$ and $w_{E_{b}}$ are the moisture contents at the time of testing of the small clear sample and the full pole respectively.

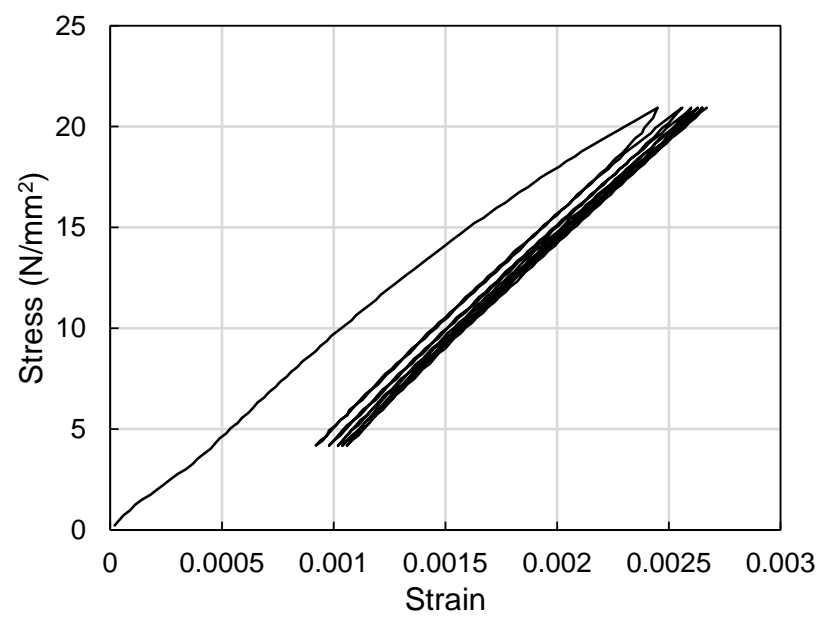

Figure 5. Typical stress-strain curve of small clear bamboo sample (pole M05)

The experimental values of $E_{c}$ obtained for all bamboo poles are shown in Table 1 and Figure 4. 3.1.4 Volume fraction, $v_{f}$ 
The volume fraction of the poles, defined in Equation (8), was calculated based on the digital processing of images taken from cross sections of four small specimen blanks adjacent to the $E_{c}$ samples. These specimens were lightly sanded with $600 / 1200$ grit silicon carbide sanding paper prior to capturing their image with a Nikon D7200 camera equipped with an AF-S DX Micro NIKKOR $85 \mathrm{~mm}$ f/3.5G ED VR lens. Figure 6 shows the original digital image and the corresponding post-processed binary one from which the area of fibres was calculated using a bespoke Matlab [28] script [10]. The volume fraction calculated neglects the contribution from the small hollow conducting vessels clustered around fibre bundles as they constitute only less than approximately $8 \%$ of the total cross-sectional area [29]. The average volume fraction calculated for each pole is shown in Table 1.

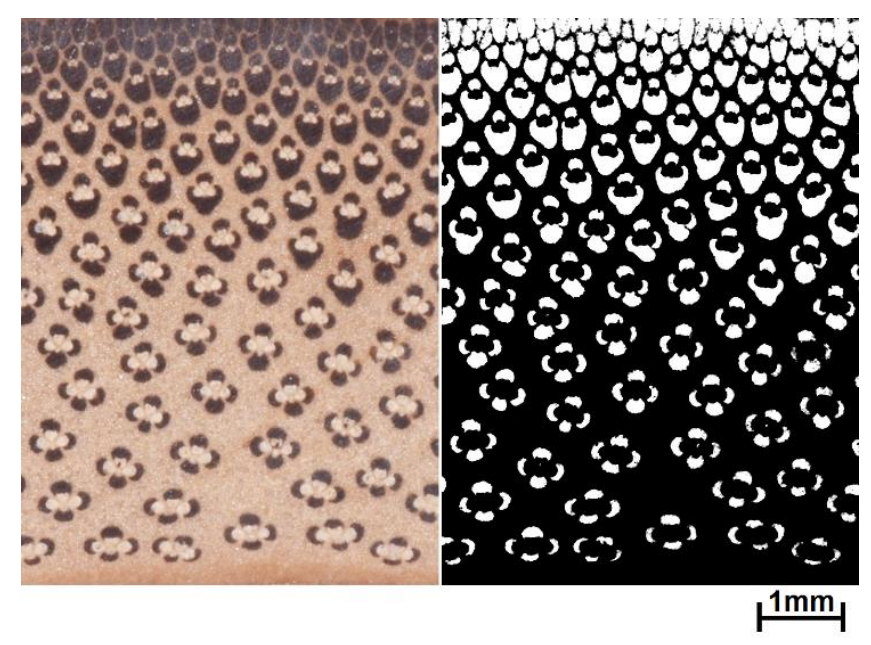

Figure 6. Digital image of bamboo wall and its corresponding binary image (pole M03)

\subsubsection{Tensile elastic modulus, $E_{t}$}

The tensile elastic modulus, $E_{t}$, was inferred from the four-point bending tests results of a control sample of two poles (M19 \& M20) instrumented with five axial strain gauges at the midspan section, with a sensitive grid of $10 \mathrm{~mm} \times 3 \mathrm{~mm}$ (length $\mathrm{x}$ width) in a quarter-bridge configuration and a resistance of $120 \Omega$. These strain gauges were installed on the front face of the poles $\left(0 \leq \alpha \leq 180^{\circ}\right.$ in Figure 1$)$ at equal $\left(45^{\circ}\right)$ intervals. The longitudinal strains measured for both poles at the maximum force, $F_{l i n}$, are shown in Figures $7 \& 8$ displaying the expected linear distribution and shift of the neutral axis towards the tension region of the cross section. The position of the neutral axis was calculated from the intercept, $b$, of the best fit lines shown in Figures $7 \& 8$ and so, the angle $\theta$ (Figure 1) can be obtained from: 
$\cos \theta=\frac{-b}{\bar{R}}$

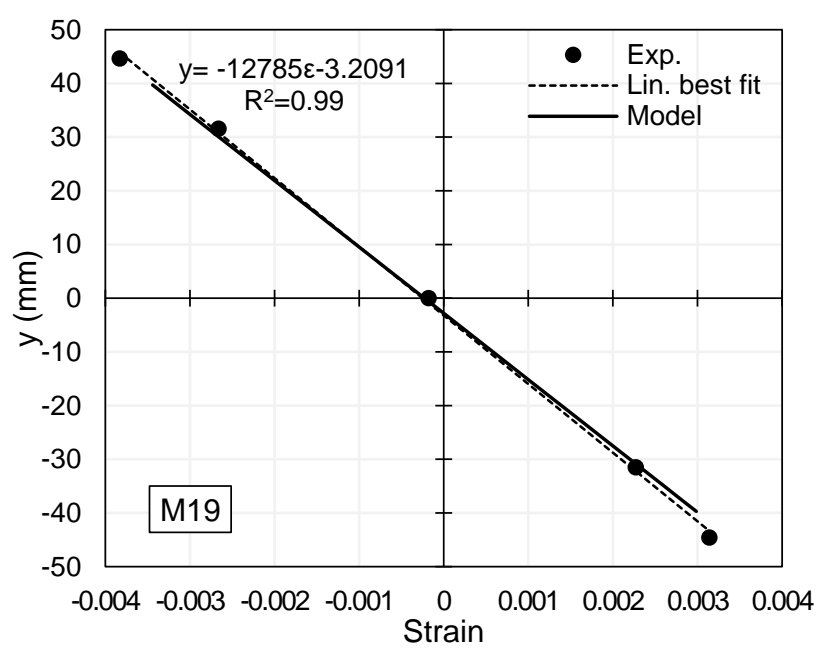

Figure 7. Analytical and experimental longitudinal strain distribution (pole M19)

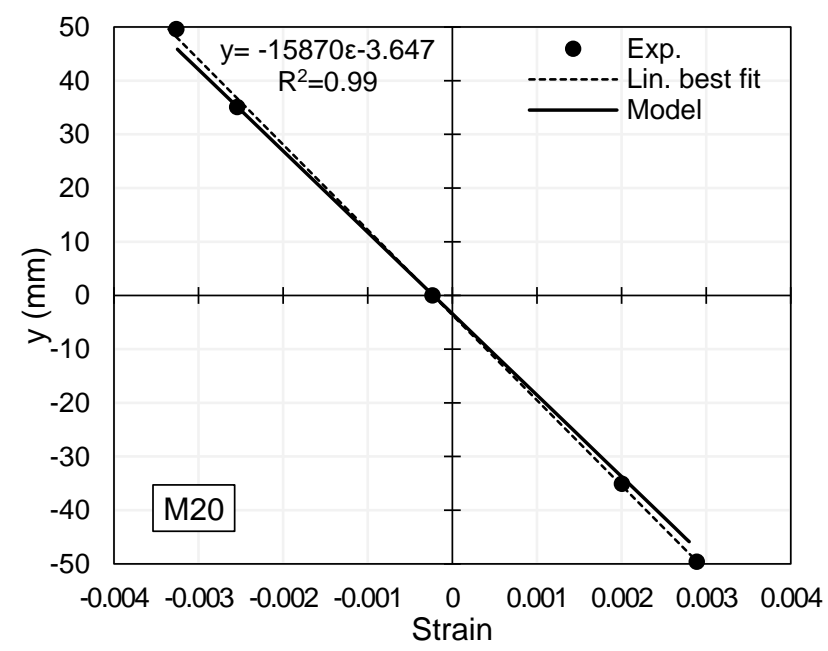

Figure 8. Analytical and experimental longitudinal strain distribution (pole M20)

Knowing the value of $\theta$, the tensile elastic modulus can be calculated from Equations (9) and (13) as:

$E_{t}=\frac{E_{c}(\theta-\tan \theta-\pi)}{v_{f}(\theta-\tan \theta)}$

The values of $E_{t}$ obtained for poles M19 and M20 were 46,440 N/mm ${ }^{2}$ and 45,740 N/mm² respectively. These values are in very good agreement with the elastic modulus of fibres of $46,000 \mathrm{~N} / \mathrm{mm}^{2}$ obtained by Amada et. al. [14] applying the rules of mixtures for composites to the experimental results of tensile tests on thin bamboo slices. Based on these findings, a constant value for $E_{t}$ of $46000 \mathrm{~N} / \mathrm{mm}^{2}$ was adopted for this study. The theoretical strain 
distributions for poles M19 and M20 shown in Figures $7 \& 8$ were obtained from Equation (2) based on the maximum tensile strain $\varepsilon_{t}$ (Figure 1) calculated from Equation (20) as:

$\varepsilon_{t}=\frac{M \bar{y}}{I K E_{t}}$

where $M$ is the maximum bending moment along the pole which, based on a clear span, $L$, of 3 $\mathrm{m}$ for all poles, is given by:

$M=\frac{F_{\text {lin }} L}{6}$

\subsection{Analytical results}

The theoretical equivalent modulus of elasticity, $E_{\text {eq }}$, for each of the 20 poles considered in this study, was calculated from Equation (17) based on the physical and mechanical properties obtained experimentally and assuming a constant tensile elastic modulus, $E_{t}$, for all poles of $46,000 \mathrm{~N} / \mathrm{mm}^{2}$.A comparison between this equivalent modulus of elasticity and the apparent modulus of elasticity, $E_{b}$, obtained from the experimental four-point bending tests is shown in Table 1 and Figure 4. There is good agreement between the analytical and experimental modulus of elasticity with an average difference of $4.5 \%$ providing a suitable level of confidence in the proposed model to quantify the cross-sectional bending strain and stress distribution in bamboo poles in bending. It is also interesting to note that these results show an average difference of $9.5 \%$ between $E_{e q}$ and the compressive elastic modulus of the small clear samples, $E_{c}$, which could be a useful parameter to adopt in preliminary conceptual designs subject to confirmation by further studies. By way of illustration, Figure 9 shows the results of the bimodulus model applied to the control pole M20 $\left(F_{\text {lin }}=4020 \mathrm{kN}, \Delta_{\text {lin }}=61.3 \mathrm{~mm}\right)$ including the theoretical strain and stress distributions under the experimental four-point bending test from Equations (2), (21), (27) and (35). For comparison, this figure also includes the strain, $\varepsilon^{\prime}$, as well as the normal, $\sigma^{\prime}$, and shear, $\tau^{\prime}$, stress distributions calculated from standard bending theory for a uniform material given by [30]:

$$
\begin{aligned}
\varepsilon^{\prime} & =\frac{\sigma^{\prime}}{E_{e q}} \\
\sigma^{\prime} & =\frac{M \bar{R} \cos \alpha}{I} \\
\tau^{\prime} & =\frac{V \sin \alpha}{\pi t \bar{R}}
\end{aligned}
$$


The strains calculated from the proposed model show a linear distribution with the expected shift of the neutral axis towards the tension region of the cross section. This shift in the neutral axis position and the significantly higher normal tensile stresses predicted by the bilinear model contribute to achieve a more accurate representation of the overall cross-sectional bending stress distribution in bamboo poles compared to that based on standard bending theory. The value of the theoretical shear stresses calculated are approximately $1 / 10^{\text {th }}$ of the mean shear capacity previously reported for clear samples of Moso [13] confirming the negligible influence of shear effects on the reported behaviour.
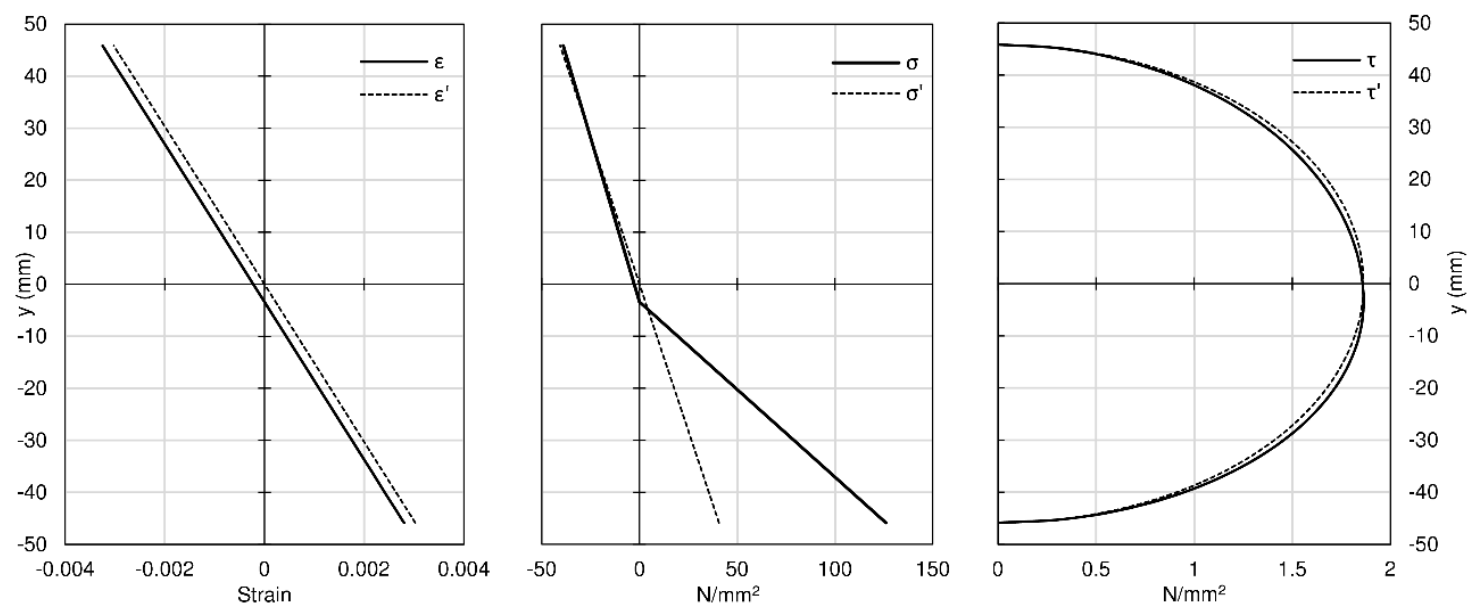

Figure 9. Theoretical strain and stress distributions from bimodulus model and standard bending theory (pole M20)

\section{Conclusions}

This paper presents the development of an analytical model to determine the elastic bending stress distribution in bamboo poles taking into account the significantly different compressive and tensile elastic moduli of the material. The experimental parameters required for the use of this bimodulus model are the compressive elastic modulus and volume fraction of small clear bamboo samples which can be experimentally determined using a low-capacity, table-top testing frame and an entry level single-lens reflex (SLR) camera. In addition, a constant value for the elastic modulus of bamboo fibres in tension of $46,000 \mathrm{~N} / \mathrm{mm}^{2}$ was indirectly determined from the experimental bending strain distribution measured on two control Moso bamboo poles. Based on these parameters, the proposed analytical model was validated comparing the predicted apparent modulus of elasticity against the corresponding modulus obtained from a 
series of four-point bending tests. The average difference between analytical and experimental results was found to be $4.5 \%$ which suggests that the proposed model is capable of predicting the elastic strain and stress distribution in bamboo poles in bending including the shift of the neutral axis towards the tensile side of the section experimentally confirmed in the control poles. However, due to the inherent variability and limited consistent historical data for different bamboo species, further experimental tests are required to validate the proposed model for other species including the applicability of adopting a constant tensile elastic modulus for their fibres.

\section{Acknowledgements}

Funding: This work was supported by the UK Engineering and Physical Sciences Research Council (EPSRC) [grant numbers: EP//M017702/1 \& EP/P510890/1]; and the British Council/CSC UK-China Joint Research and Innovation Partnership Fund [grant number: UK-276080305].

\section{References}

[1] GABC, Global status report- Towards a zero-emission, efficient and resilient buildings and construction sector, 2018 http://wedocs.unep.org/bitstream/handle/20.500.11822/27140/Global_Status_2018.pdf?s equence $=1$ \&isAllowed $=y$.

[2] UN, Transforming our world: The 2030 agenda for sustainable development, US, 2015.

[3] INBAR, Bamboo and sustainable consumption: seminar on SDG 12- INBAR, (2017). https://www.inbar.int/bamboo-and-sustainable-comsumption-seminar-on-sdg-12/ (accessed August 22, 2019).

[4] P. Van Der Lugt, Booming bamboo, International Network for Bamboo and Rattan, Beijing, China, 2017.

[5] W. Liese, Research on bamboo, Wood Sci. Technol. 21 (1987) 189-209.

[6] W. Wu, Q. Liu, Z. Zhu, Y. Shen, Managing Bamboo for Carbon Sequestration, Bamboo Stem and Bamboo Shoots, Small-Scale For. 14 (2015) 233-243. doi:10.1007/s11842014-9284-4.

[7] S. Brown, Present and potential roles of forests in the global climate change debate, Unasylva. (1996) 3-10. http://www.fao.org/docrep/w0312e/w0312e03.htm\#present and 
potential roles of forests in the global climate change debate.

[8] I.R. Hunter, Bamboo — solution to problems, J. Bamboo Ratt. 1 (2002) 101-107.

[9] P. Van der Lugt, A.A.J.F. Van den Dobbelsteen, J. Janssen, An environmental, economic and practical assessment of bamboo as a building material for supporting structures, Constr. Build. Mater. 20 (2006) 648-656. doi:10.1016/j.conbuildmat.2005.02.023.

[10] R. Lorenzo, M. Godina, L. Mimendi, H. Li, Determination of the physical and mechanical properties of moso, guadua and oldhamii bamboo assisted by robotic fabrication, J. Wood Sci. 66 (2020) 20. doi:10.1186/s10086-020-01869-0.

[11] J. Janssen, Designing and Building with Bamboo, 2000.

[12] K.A. Harries, J. Bumstead, M. Richard, D. Trujillo, Geometric and material effects on bamboo buckling behaviour, Proc. Inst. Civ. Eng. - Struct. Build. 170 (2017) 236-249. doi:10.1680/jstbu.16.00018.

[13] R. Lorenzo, M. Godina, L. Mimendi, H. Li, Determination of the physical and mechanical properties of moso, guadua and oldhamii bamboo assisted by robotic fabrication, J. Wood Sci. 66 (2020) 20. doi:10.1186/s10086-020-01869-0.

[14] S. Amada, Y. Ichikawa, T. Munekata, Y. Nagase, H. Shimizu, Fiber texture and mechanical graded structure of bamboo, Compos. Part B Eng. 28 (1997) 13-20. doi:10.1016/S1359-8368(96)00020-0.

[15] S. Amada, T. Munekata, Y. Nagase, Y. Ichikawa, A. Kirigai, Y. Zhifei, The Mechanical Structures of Bamboos in Viewpoint of Functionally Gradient and Composite Materials J. Compos. Mater. 30 (1996) 800-819. doi:10.1177/002199839603000703.

[16] F. Nogata, H. Takahashi, Intelligent functionally graded material: Bamboo, Compos. Eng. 5 (1995) 743-751. doi:10.1016/0961-9526(95)00037-N.

[17] BSI ISO 22157, British adoption for International Standard Organization ISO 22157: 2019 Bamboo structures - Determination of physical and mechanical properties of bamboo culms - Test methods, (2019) 25. https://www.iso.org/standard/65950.html.

[18] P.G. Dixon, L.J. Gibson, The structure and mechanics of Moso bamboo material, J. R. Soc. Interface. 11 (2014). doi:10.1098/rsif.2014.0321. 
[19] Z.P. Shao, C.H. Fang, S.X. Huang, G.L. Tian, Tensile properties of Moso bamboo (Phyllostachys pubescens) and its components with respect to its fiber-reinforced composite structure, Wood Sci. Technol. 44 (2010) 655-666. doi:10.1007/s00226-0090290-1.

[20] X. Zhang, J. Li, Z. Yu, Y. Yu, H. Wang, Compressive failure mechanism and buckling analysis of the graded hierarchical bamboo structure, J. Mater. Sci. 52 (2017) 69997007. doi:10.1007/s10853-017-0933-9.

[21] S. Timoshenko, Strength of materials, D. Van Nostrand Comany Inc, New York, USA, 1930.

[22] F. Tabaddor, Analysis for beams made of bi-modulus elastic orthotropic materials, Fibre Sci. Technol. 9 (1976) 51-62. doi:10.1016/0015-0568(76)90023-3.

[23] F. Tabaddor, Constitutive equations for bimodulus elastic materials, AIAA J. 10 (1972) 516-518. doi:10.2514/3.50131.

[24] A.E. Green, J.Z. Mkrtichian, Elastic solids with different moduli in tension and compression, J. Elast. 7 (1977) 369-386. doi:10.1007/BF00041729.

[25] R.C. Hibbeler, Mechanics of materials, 10th ed., Pearson Education Inc, 2017.

[26] Delmhorst BD-2100, Moisture Meters, (2019). https://www.delmhorst.com/moisturemeters/bd-2100 (accessed January 24, 2019).

[27] MCBI, Ministry of Construction and Building Industry. MCBI: JG/t 199: 2007. Testing methods for the physical and mechanical properties of bamboo materials used in construction industry, (2007) 1-47.

[28] MathWorks, Matlab and Image Processing Toolbox release 2017b, (2017).

[29] W. Liese, The anatomy of bamboo poles, International Network for Bamboo and Rattan, Beijing, China, 1998.

[30] W. Young, R.G. Budynas, Roark's formulas for stress and strain, Seventh Ed, McGRawHill, US, 2002. 